\title{
Genotype-Guided Prescription of Azathioprine Reduces the Incidence of Adverse Drug Reactions in TPMT Intermediate Metabolizers to a Similar Incidence as Normal Metabolizers
}

\author{
Ana Casajús · Pablo Zubiaur · Marta Méndez • Diana Campodónico · Antía Gómez • Marcos Navares-Gómez • \\ Gonzalo Villapalos-García • Paula Soria-Chacartegui · Jesús Novalbos • Manuel Román · Gina Mejía-Abril • \\ Dolores Ochoa · Francisco Abad-Santos
}

Received: November 25, 2021 / Accepted: January 27, 2022 / Published online: February 22, 2022

(C) The Author(s) 2022

\begin{abstract}
Introduction: Thiopurine drugs are purine nucleoside analogues used for treatment of different immune-related conditions. To date, different studies highlighted the importance of thiopurine methyltransferase (TPMT) genotyping in patients who initiate treatment with thiopurines to make an adequate dose adjustment. We aimed to investigate the influence of TPMT phenotype, concomitant treatments, and demographic characteristics on the incidence of
\end{abstract}

A. Casajús · P. Zubiaur · M. Méndez

D. Campodónico · A. Gómez · M. Navares-Gómez .

G. Villapalos-García · P. Soria-Chacartegui ·

J. Novalbos · M. Román · G. Mejía-Abril ·

D. Ochoa · F. Abad-Santos $(\bowtie)$

Clinical Pharmacology Department, Hospital Universitario de La Princesa, Instituto Teófilo Hernando, Universidad Autónoma de Madrid (UAM), Instituto de Investigación Sanitaria La Princesa (IP), C/ Diego de León, 62, 28006 Madrid, Spain

e-mail: francisco.abad@uam.es

P. Zubiaur · G. Mejía-Abril · D. Ochoa .

F. Abad-Santos

UICEC Hospital Universitario de La Princesa, Plataforma SCReN (Spanish Clinical Research Network), Instituto de Investigación Sanitaria La Princesa (IP), Madrid, Spain

P. Zubiaur · F. Abad-Santos

Centro de Investigación Biomédica en Red de Enfermedades Hepáticas y Digestivas (CIBERehd), Instituto de Salud Carlos III, Madrid, Spain adverse reactions (ADRs) in patients who start treatment with azathioprine (AZA).

Methods: This was an observational and retrospective study. The study population comprised 109 patients who started treatment with AZA following routine TPMT genotyping before June 2019 and who were routinely followed up at Hospital Universitario de La Princesa. The incidence of ADRs and treatment duration were evaluated according to TPMT phenotype.

Results: Forty-five men and 64 women were recruited, with a mean age of $67.6 \pm 18.5$. The medical specialty with the most requests was dermatology (45.9\%) and the most frequent disease for which genotyping was requested was bullous pemphigoid (27.5\%). All patients were normal metabolizers (NM), except for eight intermediate metabolizers (IM) $(7.3 \%)$; no poor metabolizers (PM) were found. The initial azathioprine dose was subtherapeutic in both groups $(103.2 \pm 45.4 \mathrm{mg}$ in NMs and $75 \pm 32.3 \mathrm{mg}$ in IMs), increasing during the first months of treatment, especially in NMs $(120.3 \pm 41.3$ vs. $78.6 \pm 30.4 \mathrm{mg}$ in IMs, $p=0.011)$. Most patients $(73.4 \%)$ received corticosteroids to keep the disease under control; and for $41.2 \%$ of NMs, physicians were able to reduce the dose at 6 months post treatment. No IMs completed 6 months of treatment. Hepatotoxicity, gastric intolerance, and blood disorders were the most common ADRs. The incidence of ADRs in the sample was $28.4 \%$ $(n=31)$ with a similar trend between IMs 
(37.5\%) and NMs (27.8\%). Patients undergoing concomitant treatment with allopurinol were associated with a higher incidence of ADRs ( $n=4,100 \%$ vs. $n=105,20 \% ; p=0.002$ ).

Conclusion: TPMT genotyping before AZA prescription reduces ADR incidence in IMs to a similar level as NMs in the Spanish population. However, it is important to note no IMs completed 6 months of treatment, suggesting that there may be some differences in drug tolerability according to phenotype. In addition, most NMs are treated with subtherapeutic doses, are poorly followed up, and thus suffer avoidable ADRs. Finally, concomitant therapies that inhibit the xanthine oxidase enzyme $(\mathrm{XDH})$, such as allopurinol, predispose to ADRs. Therefore, pharmacogenetic testing should be integrated as an additional clinical tool, in such a way that each patient receives personalized, precision treatment, where all factors influencing drug response are considered.

Keywords: Adverse drug Azathioprine; Genotyping; Precision medicine; TPMT

\section{Key Summary Points}

Adverse reaction (ADR) rate in thiopurine methyltransferase (TPMT) intermediate metabolizers (IMs) was similar to that of normal metabolizers (NMs) after dose adjustments

In contrast, treatment duration in TPMT IMs was significantly shorter in NMs compared to NMs, which suggests that, despite showing a similar ADR rate, drug tolerability may be worse in IMs

Xanthine oxidase inhibitors such as allopurinol increase the risk for ADRs; therapy must be guided and adjusted accordingly if these medications are used concomitantly

Close monitoring of the patient is crucial to detect the appearance of early ADRs and avoid them as far as possible

\section{INTRODUCTION}

Thiopurine drugs (azathioprine [AZA], 6-mercaptopurine [6-MP], and 6-thioguanine [6-TG]) are purine nucleoside analogues, which trigger cell death [1]. These drugs are widely used for the treatment of blood disorders such as leukemia (e.g., acute lymphoblastic and acute myeloid leukemia), mainly in children; and for the treatment of autoimmune disorders (e.g., Crohn's disease and rheumatoid arthritis, among others). Furthermore, they are used as immunosuppressants in organ transplant recipients [2].

As inactive prodrugs, they require intracellular activation, catalyzed by multiple enzymes, to exert cytotoxicity. AZA is transformed by glutathione-S-transferase (GST) enzymes to 6-MP extracellularly and a small amount of the latter becomes thiouric acid (6-TUA) through the action of xanthine oxidase enzyme (XDH); 6-TUA is then degraded. The remaining 6-MP enters the cell through SLC transporters where two intracellular enzymes, i.e., hypoxanthine phosphoribosyltransferase (HPRT) and thiopurine methyltransferase (TPMT), compete to break down 6-MP into various metabolites (Fig. 1) [2]. HPRT metabolizes 6-MP to thioinosine monophosphate (TIMP); the pathway then branches, and dozens of metabolites are produced through the action of several enzymes, including nudix hydrolase 15 (NUDT15) and TPMT. Eventually, the following active metabolites are formed: firstly, methylthioinosine monophosphate (MeTIMP) and methylmercaptopurine ribonucleotide (MeMPR) are formed which are responsible for inhibiting the de novo purine synthesis and hepatotoxicity. Secondly, thioguanine nucleotide triphosphate (TGTP) is formed, which is incorporated into RNA and causes apoptosis and inhibits Rac1, leading to apoptosis of activated T cells; finally, 6-thiodeoxydeoxyguanosine trisphosphate (TdGTP) is formed which is incorporated into DNA causing damage and apoptosis (Fig. 1) [3].

Furthermore, TPMT transforms 6-MP into 6-methylmercaptopurine (MeMP) and NUDT15 transforms TdGTP into TdGMP, which are 


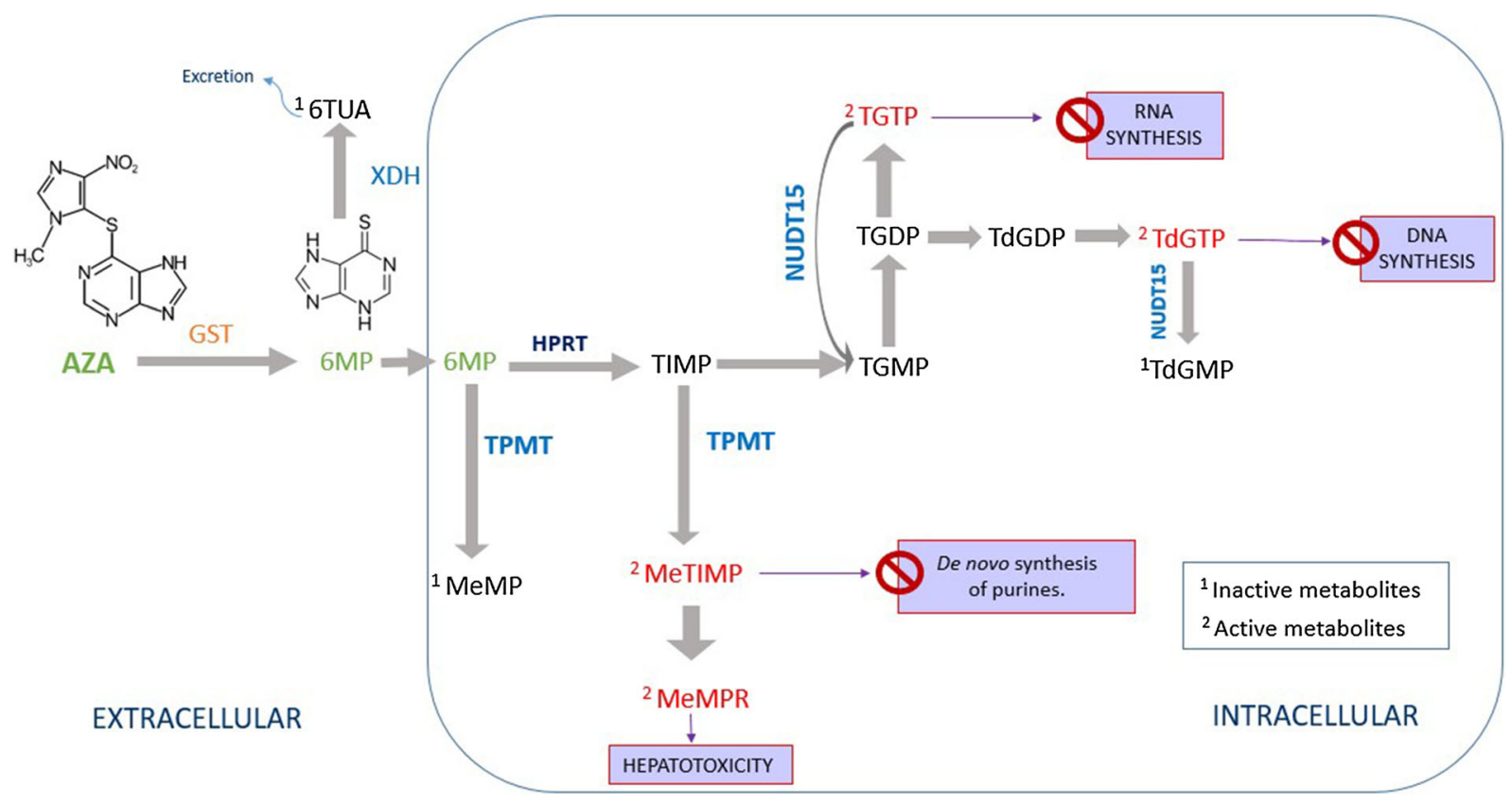

Fig. 1 Thiopurine metabolic pathway mediated by TPMT and NUDT15. $A Z A$ azathioprine, GST glutathione- $S$ transferase, 6-MP 6-mercaptopurine, $X D H$ xanthine oxidase enzyme, 6TUA thiouric acid, HPRT hypoxanthine phosphoribosyltransferase, TIMP thioinosine monophosphate, TPMT thiopurine methyltransferase, MeMP methylmercaptopurine, MeTIMP methylthioinosine

considered the main inactivating metabolic route of thiopurines. Therefore, TPMT and NUDT15 enzyme activity is inversely related to active metabolite levels and therefore to drug toxicity.

TPMT polymorphisms are related to enzymatic function variability [4]. Individuals who inherit two no-function TPMT alleles (i.e., poor metabolizers $[\mathrm{PM}]$ ) are associated with a significantly higher risk for adverse drug reactions (ADRs) as active metabolites accumulate at standard thiopurine doses. Despite exhibiting higher active metabolite levels than normal metabolizers (NM), about $40-70 \%$ of TPMT intermediate metabolizers (IM) tolerate standard thiopurine doses [5], but still the incidence of ADRs is much higher than in NMs (around $79 \%$ vs. $35 \%$ [6]). The most frequent ADRs caused by thiopurine drugs are hepatotoxicity, digestive intolerance, and myelotoxicity (with leukopenia being of major concern), the last of monophosphate, $M e M P R$ methylmercaptopurine ribonucleotide, TGMP thioguanosine monophosphate, TGDP thioguanine nucleotide diphosphate, TGTP thioguanine nucleotide triphosphate, TdGMP thiodeoxydeoxyguanosine monophosphate, TdGDP 6-thiodeoxydeoxyguanosine bisphosphate, TdGTP 6-thiodeoxydeoxyguanosine trisphosphate

these being the most common and life-threatening ADR produced by these drugs $[1,7]$.

In March 2011, the Clinical Pharmacogenetics Implementation Consortium (CPIC) published their guideline on thiopurine prescription and TPMT testing [8], which was updated in October 2018 including NUDT15 testing [9]. Briefly, a 30-80\% reduction in the starting dose is recommended for NUDT15 or TPMT IMs while NMs should start with normal starting dose; additional dose adjustments may be done on the basis of disease-specific guidelines. PMs should not receive thiopurines for indications other than malignant conditions; for the latter, drastically reduced doses (reduce daily dose by tenfold and dose thrice weekly) should be prescribed [9].

Since 2006, the Pharmacogenetics Unit of Hospital Universitario de La Princesa has performed the determination of TPMT polymorphisms to help guide thiopurine prescription 
[10]. Until June 2019, around 643 patients had been analyzed.

Although there is evidence that dose adjustment in these patients leads to a higher safety profile, the full set of clinical implications of pharmacogenetic testing implementation remains to be described in the Spanish population. In this work, we aimed to describe the effects of preemptive TPMT genotyping on (a) ADR incidence between NMs and other phenotypes and (b) the treatment duration with AZA in the population. The present work is part of the La Princesa Multidisciplinary Initiative for the Implementation of Pharmacogenetics (PriME-PGx) [10].

\section{METHODS}

\section{Study Design and Population}

This was an observational and retrospective study that aimed to evaluate whether TPMT genotype-based AZA prescription reduced the risk for ADRs in TPMT IMs or PMs patients to a comparable level to that of NMs. Furthermore, the required duration of treatment with AZA was evaluated. A total of 643 patients over 18 years old who had been genotyped for TPMT between February 2006 and June 2019 in our hospital were considered for inclusion in the study. Inclusion criteria were as follows: patients with TPMT genotyping prior to initiating treatment with AZA and routinely followed up at Hospital Universitario de La Princesa. Exclusion criteria were as follows: patients who, prior to treatment with AZA, had a leukocyte count less than $2500 / \mathrm{mm}^{3}$ or patients receiving other immunosuppressive concomitant treatments.

The study was submitted to the Independent Ethics Committee at Hospital Universitario de La Princesa which approved the study, with registration number 3999 and authorized the waiver on the request for informed consent as patients had previously consented the test as part of their routine medical care and data was collected from patients' electronic medical records. Research was conducted complying with the revised Declaration of Helsinki and Spanish Biomedical Law.

\section{Genotyping}

DNA was extracted from peripheral blood stored in an EDTA $\mathrm{K}_{2}$ tube using a MagNa Pure System (Roche Applied Science, USA) and Maxwell ${ }^{\circledR}$ RSC Automated DNA extractor (Promega Biotech Iberica S.L). DNA purity (the ratio of absorbance at $260 \mathrm{~nm}$ and $280 \mathrm{~nm}$ ) and concentration were assessed with a NanoDrop ND1000 instrument (Wilmington, DE). A LightCycler 2.0 Real Time thermal cycler (Roche Diagnostics, Mannheim, Germany) was used to genotype TPMT*2 (rs1800462), *3A (rs1800460, rs1142345), *3B (rs1800460), *3C (rs1142345) alleles.

LightSNP or Light-MIX_probes (Roche Diagnostics, Mannheim, Germany) designed by TIB MOLBIOL (Berlin, Germany) were used following the manufacturer's instructions. The genotyping analysis was carried out with the LightCycler Software, v.4.1, based on melting curves.

\section{Study Variables}

The main dependent variables of the study were the incidence of ADRs and the treatment duration with AZA. As secondary variables, the following ones were evaluated: blood disorders, including the incidence of leukopenia (defined as a white blood cell count lower than 2500 cells $/ \mathrm{mm}^{3}$ ), clinically defined hepatotoxicity and gastric intolerance. As independent variables, the following were considered: TPMT phenotype, sex, age, tobacco consumption, coexistent cardiovascular diseases (diabetes, dyslipidemia, hypertension), concomitant drugs that affect TMPT activity (e.g., corticosteroids, allopurinol and salicylates), AZA dose, evidence of close patient monitoring [e.g., complete blood cell counts (CBCs), liver chemistries, platelet count (PC) and creatinine clearance].

To retrieve clinical variables, patient medical records and databases at Hospital Universitario de La Princesa in Madrid were reviewed, 
without modifying data in the information systems and without involving direct contact with the patients to obtain additional information.

\section{Statistical Analysis}

Cross tables were used to describe the incidence of categorical variables according to another categorical variable (e.g., TPMT phenotype according to sex). For statistical inference, a Chi-squared or a Fisher's exact test were used and adjusted the significance following Bonferroni correction for multiple comparisons. For the comparison of means according to categorical variables (e.g., the leukocyte count according to tobacco consumption), a $t$-test (two categories within a variable) or an ANOVA test (more than two categories within a variable) were used. Data analysis was performed using the SPSS statistical package, version 23.0. The significance level for all hypothesis contrast tests was $p<0.05$.

\section{RESULTS}

The final sample consisted of 109 patients who met all inclusion criteria and no exclusion criteria. In the initial sample of 643 patients, 204 were not followed up at our hospital, $121 \mathrm{did}$ not initiate treatment with AZA, 107 did not have available information in their medical records, 100 were not genotyped prior to initiating treatment, and two were on other immunosuppressive therapies.

Among our study population, a greater prevalence of women was observed (Table 1). Women had similar age and lower weight than men; smoking habit was similar according to sex (Table 1). Several patients presented cardiovascular risk factors with a higher prevalence of arterial hypertension followed by dyslipidemia and diabetes (Table 1). The medical specialty that most frequently requested TPMT genotyping was dermatology (Table 1). The disease for which genotyping was most requested was bullous pemphigoid being more frequent in men than women (Table 1).
Eight ${ }^{*} 1 /{ }^{*} 3 A$ (IMs) patients were found $(7.3 \%)$ of which three were women and five were men, while the remaining 101 carried the $T_{P M T}{ }^{*} 1{ }^{*} 1$ genotype (NMs) $(92.7 \%)$. No PMs were identified.

IMs began with a mean (SD) dose of AZA of $75 \mathrm{mg}$ (32.3) compared to NMs, who started with $103.2 \mathrm{mg} \quad(45.4) \quad(p=0.111) \quad$ (Table 2, Fig. 2). IMs required no further reduction of the initial dose in any case. Conversely, $16 \mathrm{NMs}$ $(24.2 \%)$ required dose reductions $(p=0.140)$ and 35 NMs (41.5\%) and 1 IM (12.5\%) required a dose increase $(p=0.111)$. Overall, $51 \mathrm{NMs}$ $(50.5 \%)$ required dose changes during treatment, while only one IM subject (12.5\%) required a dose adjustment $(p=0.038)$. The final dose for IMs was $78.6 \mathrm{mg}$ (30.4) and for NMs it was $120.3 \mathrm{mg}$ (41.3), with a higher final dose in NMs than in IMs $(p=0.011)$ (Fig. 2, Table 2).

Regarding concomitant therapies, most patients $(73.4 \%)$ were undergoing treatment with systemic corticosteroids (Table 2). At 6 months post treatment, 33 patients $(41.2 \%)$, all of them NMs, were able to reduce and even suppress in some cases the corticosteroid dose. This variable was not collected for IMs as none of them completed the 6-month treatment.

For 63 NMs (62.4\%), treatment duration was at least 6 months while no IMs exceeded a 6 -month treatment duration $(p=0.032)$. On the one hand, of the eight IMs, one of them discontinued treatment because of blood disorders, one because of hepatotoxicity, another one because of gastric intolerance, one patient died of an unrelated cause, and for the other four no data were available on the reason for treatment discontinuation. On the other hand, of the 38 NMs who stopped treatment, 7 suffered digestive intolerance, 6 hepatotoxicity, 4 blood disorders, 5 discontinued therapy on their own decision, 2 because of lack of improvement with treatment and 3 patients died as a result of non-drug-related causes; the remaining 11 causes of treatment withdrawal were not collected.

The incidence of ADRs in the sample was $28.4 \%(n=31)$ with a similar trend between NMs and IMs $(27.8 \%$ vs. $37.5 \%, p=0.309)$ (Table 3). The three observed ADRs were 
Table 1 Baseline characteristics of the study population

\begin{tabular}{|c|c|c|c|c|}
\hline & Total & Male & Female & $p$ \\
\hline$N$ & $109(100 \%)$ & $45(41.3 \%)$ & $64(58.7 \%)$ & \\
\hline Age (years) & $67.6(18.5)$ & $68.1(18.1)$ & $67.2(18.9)$ & 0.796 \\
\hline Weight (kg) & $71.7(16.1)$ & $80.3(11.6)$ & $62.5(15.4)$ & 0.001 \\
\hline Smokers & $28(25.7 \%)$ & $14(31.1 \%)$ & $14(21.9 \%)$ & 0.277 \\
\hline \multicolumn{5}{|l|}{ Cardiovascular risk factors } \\
\hline Hypertension & $39(35.8 \%)$ & $19(42.2 \%)$ & $20(31.3 \%)$ & 0.239 \\
\hline Dyslipidemia & $36(33.0 \%)$ & $14(31.1 \%)$ & $22(34.4 \%)$ & 0.721 \\
\hline Diabetes & $24(22.0 \%)$ & $12(26.7 \%)$ & $12(18.8 \%)$ & 0.326 \\
\hline \multicolumn{5}{|l|}{ Petitionary medical specialties } \\
\hline Dermatology & $50(45.9 \%)$ & $24(53.3 \%)$ & $26(40.6 \%)$ & 0.190 \\
\hline Rheumatology & $20(18.3 \%)$ & $2(4.4 \%)$ & $18(28.1 \%)$ & 0.002 \\
\hline Pneumology & $13(11.9 \%)$ & $7(15.6 \%)$ & $6(9.4 \%)$ & 0.327 \\
\hline Neurology & $11(10.1 \%)$ & $4(8.9 \%)$ & $7(10.9 \%)$ & 0.727 \\
\hline Gastroenterology & $7(6.4 \%)$ & $4(8.9 \%)$ & $3(4.7 \%)$ & 0.378 \\
\hline Other medical specialties ${ }^{\mathrm{a}}$ & $8(7.3 \%)$ & $4(8.9 \%)$ & $4(6.3 \%)$ & 0.603 \\
\hline \multicolumn{5}{|l|}{ Indications for thiopurine prescription } \\
\hline Bullous pemphigoid & $30(27.5 \%)$ & $17(37.8 \%)$ & $13(20.3 \%)$ & 0.044 \\
\hline Vasculitis & $14(12.8 \%)$ & $2(4.4 \%)$ & $12(18.8 \%)$ & 0.028 \\
\hline Interstitial lung diseases & $13(11.9 \%)$ & $7(15.6 \%)$ & $6(9.4 \%)$ & 0.327 \\
\hline Myasthenia gravis & $10(9.2 \%)$ & $5(11.1 \%)$ & $5(7.8 \%)$ & 0.557 \\
\hline Atopic dermatitis & $10(9.2 \%)$ & $5(11.1 \%)$ & $5(7.8 \%)$ & 0.557 \\
\hline Dermatomyositis and polymyositis & $9(8.3 \%)$ & $1(2.2 \%)$ & $8(12.5 \%)$ & 0.055 \\
\hline Systemic lupus erythematosus & $7(6.4 \%)$ & $1(2.2 \%)$ & $6(9.4 \%)$ & 0.134 \\
\hline Inflammatory bowel disease (IBD) & $6(5.5 \%)$ & $4(6.3 \%)$ & $2(4.4 \%)$ & 0.194 \\
\hline Lichen & $6(5.5 \%)$ & $2(4.4 \%)$ & $4(6.3 \%)$ & 0.684 \\
\hline Other diseases ${ }^{\mathrm{b}}$ & $4(3.7 \%)$ & $1(2.2 \%)$ & $3(4.7 \%)$ & 0.500 \\
\hline
\end{tabular}

Data are shown as mean (standard deviation) or count (\%)

${ }^{a}$ Other medical specialties include emergency medicine, ophthalmology, intensive care medicine

${ }^{\mathrm{b}}$ Other diseases include autoimmune hepatitis, erythema nodosum, and scleritis

hepatotoxicity $(n=11)$, gastric intolerance $(n=10), \quad$ and blood disorders $(n=10)$ (leukopenia being the most frequent within the last of these ADRs $[n=6])$, all with a similar incidence between phenotypes (Table 3). All patients concomitantly treated with allopurinol suffered ADRs $(n=4,100 \%)$ vs. $20 \%$ of those that did not receive it $(n=105, p=0.002)$ with 
Table 2 Medication received by patients according to TPMT phenotype

\begin{tabular}{lcccc}
\hline & Total $(\boldsymbol{n}=\mathbf{1 0 9})$ & NMs $(\boldsymbol{n}=\mathbf{1 0 1})$ & IMs $(\boldsymbol{n}=\mathbf{8})$ & $\boldsymbol{p}$ \\
\hline Allopurinol & $4(3.7 \%)$ & $4(4.0 \%)$ & $0(0.0 \%)$ & 0.566 \\
Salicylates & $13(11.9 \%)$ & $12(11.9 \%)$ & $1(12.5 \%)$ & 0.959 \\
Corticosteroids & $80(73.4 \%)$ & $75(74.3 \%)$ & $5(62.5 \%)$ & 0.469 \\
Initial AZA dose (mg) & $101.3(45.1)$ & $103.2(45.4)$ & $75(32.3)$ & 0.111 \\
Final AZA dose $(\mathrm{mg})$ & $117.6(42.2)$ & $120.3(41.3)$ & $78.6(30.4)$ & $0.011^{*}$ \\
\hline
\end{tabular}

Data are shown as mean (standard deviation) or count (\%)

$A Z A$ azathioprine, $N M$ normal metabolizers, $I M s$ intermediate metabolizers, ${ }^{*} p<0.05$.

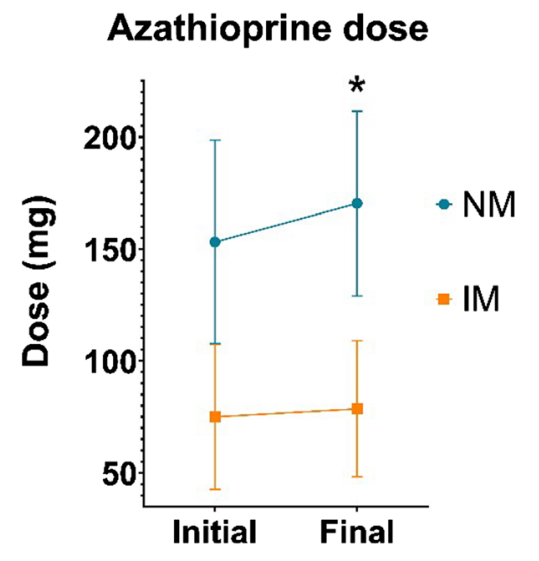

Fig. 2 Initial vs. final AZA dose according to TPMT phenotype. $N M$ normal metabolizers, IMs intermediate metabolizers, ${ }^{*} p<0.05$

blood disorders being the most frequent ADR $(75 \%, p=0.002)$. A complete CBC and $\mathrm{PC}$ at the first week of treatment was requested for most patients, but none of them was closely monitored (i.e., weekly monitoring of $\mathrm{CBC}$ and $\mathrm{PC}$ during the first month).

\section{DISCUSSION}

In the treatment with thiopurine drugs, large inter-individual differences occur as a result of genetic polymorphisms on the TPMT gene [5]. The prescribed dose is also of great relevance, as ADRs may appear because of overexposure to the drug, which may result in early withdrawal of treatment. On the other hand, an adequate dose can significantly improve quality of life of patients by keeping their disease under control; consequently, corticoid demand would be lower (which is the most commonly used concomitant drug for these pathologies), dose would be reduced and so would the incidence of ADRs. In addition, different studies recommend

Table 3 Incidence of adverse drug reactions according to TPMT phenotype

\begin{tabular}{lllll}
\hline & Total & NMs & IMs & $\boldsymbol{p}$ \\
\hline Hepatotoxicity & $11(10.1 \%)$ & $10(9.9 \%)$ & $1(12.5 \%)$ & 0.814 \\
Gastric intolerance & $10(9.2 \%)$ & $9(8.9 \%)$ & $1(12.5 \%)$ & 0.735 \\
Blood disorders & $10(9.2 \%)$ & $9(8.9 \%)$ & $1(12.5 \%)$ & 0.735 \\
Leukopenia $^{a}$ & $6(5.5 \%)$ & $5(4.6 \%)$ & $1(12.5 \%)$ & 0.367 \\
Total $^{\text {Tot }}$ & $31(28.4 \%)$ & $28(27.8 \%)$ & $3(37.5 \%)$ & 0.309 \\
\hline
\end{tabular}

$N M s$ normal metabolizers, IMs intermediate metabolizers, ADRs adverse reactions

${ }^{\mathrm{a}}$ Leukopenia is a blood disorder that was analyzed separately. 
searching for biomarkers that could help predict response and tolerability to treatment with thiopurines and therefore adapt treatment dose to the patient's genotype [11].

Allelic variants in the TPMT gene responsible for decreased or no enzyme function were identified to date in different ethnic groups. The most relevant TPMT no-function alleles in Caucasian population, by prevalence and functional impact, are $T P M T^{*} 2,{ }^{*} 3 A,{ }^{*} 3 B$, and ${ }^{*} 3 C$. These alleles account for $80-95 \%$ of patients with IM or PM phenotypes [5] with TPMT*3A being the most frequent of all in Caucasians. This is consistent with the findings of the present study, where $7.3 \%$ of IMs were identified, all of them with the $* 3 A$ allele. The most common genotype for the TPMT gene is the ${ }^{*} 1 /{ }^{*} 1$ $(\mathrm{NM})$, which, according to several studies, comprises $85-95 \%$ of the Caucasian population, followed by approximately $10 \%$ of IMs and $0.3 \%$ of PMs $[12,13]$. In our study population of 109 patients, consistent results were obtained, although no PMs were found as a result of the small sample size.

However, while TPMT genotyping makes a valuable contribution to the identification of patients at increased risk for developing ADRs, additional monitoring of patients on AZA treatment is advised [14]. For this purpose, it is recommended to perform routine analyses that consider CBC, liver chemistries, PC, and creatinine clearance. Some authors recommend weekly monitoring of $\mathrm{CBC}$ and $\mathrm{PC}$ during the first month of thiopurine treatment, followed by a biweekly follow-up during the second and third month and monthly checks thereafter [5]. Furthermore, liver function tests should be performed at 3-monthly intervals [5]. In our study population, as mentioned in the "Results", patients were not followed closely, which may have led to the appearance of more ADRs.

Different studies associated TPMT IM or PM phenotypes with a high incidence of ADRs produced by thiopurines (79\%) [6] including hepatotoxicity, gastric intolerance, and myelotoxicity [15]. In the present study, the rate of ADRs with AZA in IM patients was much lower and comparable to that of NMs $(37.5 \%$ vs. $27.8 \%$ ), due to early dose adjustment, but the risk of ADRs was not completely eliminated. In fact, no IM finished the 6-month follow-up period, which suggests that toxicity still occurs within this group of patients even after dose reduction. The small sample size probably prevents us from finding significant results; there would likely be significant results with a larger sample size, which would be consistent with the time of treatment. Furthermore, NMs are assumed to require less attention after drug prescription. However, on the basis of our results, this may not be appropriate: they still suffer considerable ADRs (27.8\%), which results in a significant impact on their quality of life and increases health care costs. Moreover, not all ADRs are explained by the patient's genotype and, therefore, genotyping prior to the start of azathioprine treatment should be followed by close patient monitoring and in no case should it replace the latter [15].

In addition, all patients that were undergoing concomitant therapy with allopurinol, i.e, all NMs, suffered ADRs. When prescribing AZA, it is important to identify patients undergoing concomitant therapies that may interact with AZA metabolism to avoid the appearance or worsening of ADRs. Special care is warranted with xanthine oxidase inhibitors when prescribing AZA; AZA dose should be reduced to a quarter of the usual dose because allopurinol, oxypurinol, and thiopurinol reduce its metabolism [16]. Once again, close monitoring of all patients may likely reduce ADR incidence; efforts are therefore warranted to teach and convince physicians to manage their patients accordingly.

Interestingly, NMs required a mean dose increase of $17 \%$. It could be proposed that the starting dose was subtherapeutic in many cases resulting in inadequate disease control. We must consider that the recommended dose is $2.5 \mathrm{mg} / \mathrm{kg}$ (around $175 \mathrm{mg} / \mathrm{day}$ ). Therefore, close monitoring of NMs seems key, because a fraction of patients are subtherapeutic and may not respond appropriately while another fraction of patients will suffer ADRs and require dose reductions. Given the clinical relevance of ADRs, the minimum effective dose should be given to reduce the risk for ADR appearance.

Finally, the treatment with AZA reduced the doses of other drugs such as corticoids. This is of 
great relevance because of the complex tolerability profile of this family of drugs. Therefore, appropriate prescription of AZA not only leads to better disease control but also reduces the demand for concomitant treatments that may also cause adverse effects, improving the overall tolerance of drug therapy.

\section{LIMITATIONS}

This study has several limitations. Firstly, NUDT15 phenotype was not considered, which is similarly a strong predictor of thiopurine dose requirements, effectiveness, and plasma levels. In contrast, NUDT15 IM or PM phenotypes are very infrequent in Caucasians and it would have been highly unlikely to find patients with a phenotype other than NM. Secondly, the exploratory nature of this work should be considered: because of the small sample size and the arbitrariness of the sample, it is possible that distributions of variables affecting the incidence of ADRs were not homogeneously distributed across phenotypes, which could lead to biased associations. Thirdly, it is a retrospective study in which data were collected from the medical records of patients for many of whom all the data was not available. It would be desirable to increase the sample size to enrich our population in IMs and PMs, which would improve the statistical power of the study.

\section{CONCLUSION}

In this study, we verified that TPMT genotyping before AZA prescription reduces ADR incidence in IMs to a similar level as NMs in the Spanish population. In contrast, despite ADR incidence not being significantly higher in the IM group, no IM completed 6 months of treatment with thiopurines compared to $67 \%$ of NMs, which suggests that IMs may still be at increased risk for toxicity even after dose adjustments. In fact, a significant fraction of IMs and NMs still suffered considerable ADRs. Moreover, 24\% of NMs required dose reductions and $41.5 \%$ required dose increases; in contrast, only one IM $(12.5 \%)$ required a dose increase. All NMs that were undergoing concomitant treatment with allopurinol suffered ADRs. Consequently, close patient follow-up to avoid the occurrence of ADRs is warranted for both IMs and NMs. In conclusion, pharmacogenetic tests should not be considered alone, but should be integrated as an additional clinical tool, in such a way that each patient receives personalized, precision treatment, where all factors influencing drug response are considered.

\section{ACKNOWLEDGEMENTS}

The authors are thankful to the participants of the study and the staff of the Pharmacogenetics Unit of Hospital Universitario de La Princesa for their effort.

Funding. Open Access funding provided thanks to the CRUE-CSIC agreement with Springer Nature. This research received no external funding. G. Villapalos-García is co-financed by Instituto de Salud Carlos III (ISCIII) and the European Social Fund (PFIS predoctoral grant, number FI20/00090). M. Navares-Gómez is financed by the ICI20/00131 Grant, Acción Estratégica en Salud 2017-2020, ISCIII. Paula Soria-Chacartegui is financed the FPI-UAM2021 predoctoral fellowship. P. Zubiaur's contract with CIBERehd is financed by the "Infraestructura de Medicina de Precisión asociada a la Ciencia y Tecnología (IMPaCT, IMP/ 00009)", Instituto de Salud Carlos III (ISCIII).

Authorship. All named authors meet the International Committee of Medical Journal Editors (ICMJE) criteria for authorship for this article, take responsibility for the integrity of the work as a whole, and have given their approval for this version to be published.

Author Contributions. AC, MM, FA-S, GM$\mathrm{A}, \mathrm{MR}$ and DO contributed to the concept and design of the manuscript. AC, DC, AG, MN-G and JN collaborated by collecting data. PZ, GVG, PS-C and AC performed the statistical analysis. Finally, AC, PZ, MM and FA-S drafted the manuscript. 
Disclosures. Dolores Ochoa, Manuel Román and Francisco Abad-Santos have been consultants or investigators in clinical trials sponsored by the following pharmaceutical companies: Abbott, Alter, Chemo, Cinfa, FAES Farma, Farmalíder, Ferrer, GlaxoSmithKline, Galenicum, Gilead, Italfarmaco, Janssen-Cilag, Kern Pharma, Normon, Novartis, Servier, Silverpharma, Teva and Zambon. Ana Casajús, Pablo Zubiaur, Marta Méndez, Diana Campodónico, Antía Gómez, Marcos Navares-Gómez, Gonzalo Villapalos-García, Paula Soria-Charcartegui, Jesús Novalbos and Gina Mejía-Abril have nothing to disclose.

Compliance with Ethics Guidelines. The study was approved by the Clinical Research Ethics Committee of Hospital Universitario de La Princesa (Madrid) and research was conducted complying with the revised Declaration of Helsinki and Spanish Biomedical Law.

Data Availability. The datasets generated during and/or analyzed during the current study are available from the corresponding author on reasonable request.

Open Access. This article is licensed under a Creative Commons Attribution-NonCommercial 4.0 International License, which permits any non-commercial use, sharing, adaptation, distribution and reproduction in any medium or format, as long as you give appropriate credit to the original author(s) and the source, provide a link to the Creative Commons licence, and indicate if changes were made. The images or other third party material in this article are included in the article's Creative Commons licence, unless indicated otherwise in a credit line to the material. If material is not included in the article's Creative Commons licence and your intended use is not permitted by statutory regulation or exceeds the permitted use, you will need to obtain permission directly from the copyright holder. To view a copy of this licence, visit http://creativecommons.org/licenses/by$\mathrm{nc} / 4.0 /$.

\section{REFERENCES}

1. Moon W, Loftus EV. Review article: recent advances in pharmacogenetics and pharmacokinetics for safe and effective thiopurine therapy in inflammatory bowel disease. Aliment Pharmacol Ther. 2016;43: 863-83.

2. Zaza G, Cheok M, Krynetskaia N, et al. Thiopurine pathway. Pharmacogenet Genom. 2010;20:573-4.

3. Peng X-X, Shi Z, Damaraju VL, et al. Up-regulation of MRP4 and down-regulation of influx transporters in human leukemic cells with acquired resistance to 6-mercaptopurine. Leuk Res. 2008;32:799-809.

4. Abaji R, Krajinovic M. Thiopurine $S$-methyltransferase polymorphisms in acute lymphoblastic leukemia, inflammatory bowel disease and autoimmune disorders: influence on treatment response. Pharmgenomics Pers Med. 2017;10: $143-56$

5. Zalizko P, Stefanovics J, Sokolovska J, et al. Thiopurine $S$-methyltransferase genetic polymorphisms in adult patients with inflammatory bowel diseases in the Latvian population. Ther Adv Gastroenterol. 2020;13:175628482093742.

6. Cabaleiro T, Roman MP, Gisbert J, Abad-Santos F. Utility of assessing thiopurine $S$-methyltransferase polymorphisms before azathioprine therapy. Curr Drug Metab. 2012;13:1277-93.

7. Dubinsky MC, Lamothe S, Yang HY, et al. Pharmacogenomics and metabolite measurement for 6-mercaptopurine therapy in inflammatory bowel disease. Gastroenterology. 2000;118:705-13.

8. Relling MV, Gardner EE, Sandborn WJ, et al. Clinical Pharmacogenetics Implementation Consortium guidelines for thiopurine methyltransferase genotype and thiopurine dosing. Clin Pharmacol Ther. 2011;89:387-91.

9. Relling MV, Schwab M, Whirl-Carrillo M, et al. Clinical Pharmacogenetics Implementation Consortium guideline for thiopurine dosing based on TPMT and NUDT 15 genotypes: 2018 update. Clin Pharmacol Ther. 2019;105:1095-105.

10. Zubiaur P, Mejía-Abril G, Navares-Gómez M, et al. PriME-PGx: La Princesa University Hospital multidisciplinary initiative for the implementation of pharmacogenetics. J Clin Med. 2021;10:3772.

11. Cornish JS, Wirthgen E, Däbritz J. Biomarkers predictive of response to thiopurine therapy in inflammatory bowel disease. Front Med. 2020;7:8. 
12. Liu C, Yang W, Pei D, et al. Genomewide approach validates thiopurine methyltransferase activity is a monogenic pharmacogenomic trait. Clin Pharmacol Ther. 2017;101:373-81.

13. Asadov C, Aliyeva G, Mustafayeva K. Thiopurine S-methyltransferase as a pharmacogenetic biomarker: significance of testing and review of major methods. Cardiovasc Hematol Agents Med Chem. 2017;15:23-30. http://www.eurekaselect.com/ 152738/article. Accessed 16 Nov 2021.

14. Yarur AJ, Abreu MT, Deshpande AR, Kerman DH, Sussman DA. Therapeutic drug monitoring in patients with inflammatory bowel disease. World J Gastroenterol. 2014;20:3475-84.

15. Ribaldone DG, Adriani A, Caviglia GP, et al. Correlation between thiopurine $S$-methyltransferase genotype and adverse events in inflammatory bowel disease patients. Medicina. 2019;55:441.

16. European Medical Agency. JAYEMPI- azathioprine drug label. 2021. https://www.ema.europa.eu/en/ documents/overview/jayempi-epar-medicineoverview_es.pdf. Accessed 13 Nov 2021. 